\title{
Risk Efficiencies of Empirical Bayes and Generalized Maximum Likelihood Estimates for Rayleigh Model under Censored Data
}

\author{
Dinesh Barot ${ }^{1}$ and Manhar Patel ${ }^{2}$ \\ ${ }^{1}$ Department of Statistics, H. L. Institute of Commerce, Ahmedabad University, Ahmedabad 380009, India \\ ${ }^{2}$ Department of Statistics, School of Sciences, Gujarat University, Ahmedabad 380009, India
}

Correspondence should be addressed to Dinesh Barot; hl.drbarot@gmail.com

Received 13 February 2014; Accepted 18 June 2014; Published 2 July 2014

Academic Editor: Shesh N. Rai

Copyright (C) 2014 D. Barot and M. Patel. This is an open access article distributed under the Creative Commons Attribution License, which permits unrestricted use, distribution, and reproduction in any medium, provided the original work is properly cited.

\begin{abstract}
The comparison of empirical Bayes and generalized maximum likelihood estimates of reliability performances is made in terms of risk efficiencies when the data are progressively Type II censored from Rayleigh distribution. The empirical Bayes estimates are obtained using an asymmetric loss function. The risk functions of the estimates and risk efficiencies are obtained under this loss function. A real data set is presented to illustrate the proposed comparison method, and the performance of the estimates is examined and compared in terms of risk efficiencies by means of Monte Carlo simulations. The simulation results indicate that the proposed empirical Bayes estimates are more preferable than the generalized maximum likelihood estimates.
\end{abstract}

\section{Introduction}

Censoring has become quite popular in life testing, reliability performances, and survival analysis. In life testing experiment, most of the time, an experimenter may be unable to observe the lifetimes of all units due to some constraints such as time, money, and resources, or it is not feasible to continue the experiment until the last observation. Censoring arises in such situations and different types of censoring can be used based on how the data are collected from the life testing experiments. The most popular censoring scheme among the various types of censoring scheme used in lifetime analysis is progressive Type II censoring scheme. Due to allowance of removal of experimental units at points other than the terminal point of an experiment, this censoring scheme is useful in many practical situations where budget constraints are in place or there is a demand for rapid testing. A significant work regarding the inference procedures based on progressive Type II censored samples has been found in the literature and journals, including Cohen [1], Balakrishnan and Aggarwala [2], Wu et al. [3], and N. W. Patel and M. N. Patel [4].
In empirical Bayes (EB) approach, an experimenter usually does not specify the unknown hyperparameter (parameter of prior distribution) but is often tempted to use some estimate of the hyperparameter. This approach is commonly used when a data-driven choice of the hyperparameter is desirable and has been described extensively by many authors such as Robbins [5], Maritz and Lwin [6], Casella [7], Carlin and Louis [8], and Lehmann and Casella [9]. Several authors, including Ali Mousa [10] and Asgharzadeh and Valiollahi [11], have made significant work regarding the inference procedures based on EB approach.

The main objective of the paper is to examine and compare the performance of the EB estimates of reliability performances relative to general entropy loss function (GELF) with that of the generalized maximum likelihood (GML) estimates in terms of risk efficiencies when the data are progressively Type II censored from the Rayleigh distribution.

The rest of the paper is organized as follows. Section 2 describes the Rayleigh model, GML estimation, EB estimation relative to GELF, risk functions of the estimates under GELF, and risk efficiencies. Section 3 considers a real life application to the wind speed data for the developed 
estimation methods and an extensive simulation study to compare the performance of EB estimates with that of GML estimates. This paper concludes with a brief discussion in Section 4 .

\section{Materials and Methods}

2.1. The Model Description. Rayleigh [12] introduced the Rayleigh distribution to describe the amplitude of sound resulting from many important sources. This distribution is widely used in communication engineering, clinical studies, life testing experiments, electrovacuum devices, reliability analysis, and applied statistics. It is also found useful to study the scattering of radiation and wind speeds or to make certain transformation. The probability density, cumulative distribution, reliability, and failure rate functions of Rayleigh distribution are given, respectively, by

$$
\begin{gathered}
f(x \mid \theta)=\frac{x}{\theta^{2}} e^{-x^{2} / 2 \theta^{2}}, \quad x>0, \theta>0, \\
F(x \mid \theta)=1-e^{-x^{2} / 2 \theta^{2}}, \\
R(t)=e^{-t^{2} / 2 \theta^{2}}, \quad t>0 \\
\lambda(t)=\frac{t}{\theta^{2}} .
\end{gathered}
$$

2.2. Likelihood Function under Progressively Type II Censored Samples. Let $n$ units be placed on a life-testing experiment, and only $m(<n)$ units are completely observed until failure. At the time of each failure occurring prior to the termination point, one or more surviving units are randomly withdrawn from the life test. Let $x_{(1)} \leq x_{(2)} \leq \cdots \leq x_{(m)}$ be the lifetimes of the $m$ completely observed units following the Rayleigh distribution (1), and $r_{1}, r_{2}, \ldots, r_{m}\left(r_{m}=n-m-\sum_{i=1}^{m-1} r_{i}\right)$ are the numbers of units withdrawn at these failure times. Then, $\underline{x}=\left(x_{(1)}, x_{(2)}, \ldots, x_{(m)}\right)$ is the progressively Type II censored sample with the censoring scheme $\underline{r}=\left(r_{1}, r_{2}, \ldots, r_{m}\right)$. The likelihood function based on $\underline{x}$ can be obtained as (see [3])

$$
L_{1}(\underline{x} \mid \theta)=\frac{A \prod_{i=1}^{m} x_{(i)}}{\theta^{2 m}} e^{-T / 2 \theta^{2}},
$$

where

$$
\begin{aligned}
& A=n\left(n-1-r_{1}\right)\left(n-2-r_{1}-r_{2}\right) \cdots\left(n-m+1-\sum_{i=1}^{m-1} r_{i}\right), \\
& T=\sum_{i=1}^{m}\left(1+r_{i}\right) x_{(i)}^{2}=\sum_{i=1}^{m}\left(n-\sum_{j=1}^{i-1} r_{j}-i+1\right)\left(x_{(i)}^{2}-x_{(i-1)}^{2}\right) .
\end{aligned}
$$

It is clear that progressive Type II censoring scheme includes complete sampling scheme $\left(r_{1}=r_{2}=\cdots=r_{m}=\right.$ $0, n=m)$ and conventional Type II right censoring scheme $\left(r_{1}=r_{2}=\cdots=r_{m-1}=0, r_{m}=n-m\right)$.

It is straightforward to show that $x_{(i)}^{2} / 2 \theta^{2}$ is distributed exponentially with mean 2 , which implies that $T$ has the gamma distribution with the shape parameter $m$, scale parameter $2 \theta^{2}$, and probability density function

$$
h(T)=\frac{e^{-T / 2 \theta^{2}} T^{m-1}}{\left(2 \theta^{2}\right)^{m} \Gamma(m)} ; \quad T>0 .
$$

2.3. Prior and Posterior Distributions. The prior distribution is an integral part of Bayesian inference, which characterizes the beliefs of the researcher before observing the results of an experiment. Suppose that the unknown scale parameter $\theta$ is the realization of a random variable which has square root inverted gamma prior with the probability density function:

$$
\pi(\theta \mid b)=\frac{\theta^{-2 b-1} e^{-1 / 2 \theta^{2}}}{\Gamma(b) 2^{b-1}} ; \quad b>0 .
$$

The prior distribution (8) has advantages over many other distributions because of its analytical tractability, richness, and easy interpretability. In light of the progressively Type II censored sample $\underline{x}$, we update the prior information about the scale parameter $\theta$ via posterior model. From Bayes' theorem, the posterior density function of $\theta$ given $\underline{x}$ can be obtained as

$$
\pi^{*}(\theta \mid \underline{x})=\frac{(1+T)^{b+m} e^{-(1+T) / 2 \theta^{2}} \theta^{-2(b+m)-1}}{2^{b+m-1} \Gamma(b+m)} .
$$

2.4. GML Estimation of Reliability Performances. The GML estimate of the parameter $\theta$, denoted by $\widehat{\theta}_{\mathrm{GML}}$, is the value of $\theta$ at which the posterior density (9) is maximal. This estimate can be identified by maximizing the log posterior density function of $\theta$; that is,

$$
\begin{aligned}
\ln \pi^{*}(\theta \mid \underline{x})= & \ln \left[\frac{(1+T)^{b+m}}{2^{b+m-1} \Gamma(b+m)}\right] \\
& -\frac{1+T}{2 \theta^{2}}-(2 b+2 m+1) \ln \theta .
\end{aligned}
$$

Differentiating (10) with respect to $\theta$, and then equalizing the obtained expression to zero, we obtain the equation

$$
\frac{1+T}{\theta^{3}}-\frac{2 b+2 m+1}{\theta}=0
$$

From (11), we have

$$
\widehat{\theta}_{\mathrm{GML}}=\sqrt{\frac{1+T}{2 b+2 m+1}} .
$$

The invariance property of GML estimation enables one to obtain the GML estimates $\widehat{R}(t)_{\mathrm{GML}}$ and $\widehat{h}(t)_{\mathrm{GML}}$ by substituting $\widehat{\theta}_{\mathrm{GML}}$ for $\theta$ in (3) and (4).

2.5. EB Estimation under GELF. Since the last couple of decades, a significant work has been developed in the field of reliability using EB approach. In this subsection, EB approach is used to derive the estimates of parameter $\theta$, reliability function $R(t)$, and failure rate function $h(t)$. In order to derive 
the EB estimates, one must specify a loss function, which represents a penalty associated with each of the possible estimates. In the Bayesian analysis, an overestimation may be more serious than an underestimation or vice versa for some estimation problems. In such situations, the use of asymmetric loss function proposed by Calabria and Pulcini [13], namely, GELF, might be appropriate. The GELF for a parameter $\phi$ with the shape parameter $\alpha(\alpha \neq 0)$ is given by

$$
L(\widehat{\phi}, \phi)=\left(\frac{\widehat{\phi}}{\phi}\right)^{\alpha}-\alpha \ln \left(\frac{\widehat{\phi}}{\phi}\right)-1
$$

The sign and magnitude of the shape parameter $\alpha$ reflect the direction and degree of asymmetry, respectively. The Bayes estimate $\widehat{\phi}_{\mathrm{GE}}$ of $\phi$ under GELF is the value $\widehat{\phi}$ that minimizes the posterior expectation of (13), and it is

$$
\widehat{\phi}_{\mathrm{GE}}=\left[E^{\pi^{*}}\left(\phi^{-\alpha} \mid \underline{x}\right)\right]^{-1 / \alpha} \text {. }
$$

The Bayes estimates of reliability performances using GELF under progressive Type II censored data are, respectively,

$$
\begin{gathered}
\widehat{\theta}_{\mathrm{GE}}=\left[\frac{\Gamma(b+m)}{\Gamma(b+m+\alpha / 2)}\right]^{1 / \alpha} \sqrt{\frac{1+T}{2}}, \\
\widehat{R}(t)_{\mathrm{GE}}=\left(1-\frac{t^{2} \alpha}{1+T}\right)^{(b+m) / \alpha}, \\
\widehat{h}(t)_{\mathrm{GE}}=\left[\frac{\Gamma(b+m)}{\Gamma(b+m-\alpha)}\right]^{1 / \alpha} \frac{2 t}{1+T} .
\end{gathered}
$$

Despite Bayes procedures that are increasingly popular, it is a common experience that expressing honest prior information about the parameter can be difficult and, in practice, one is often tempted to use some estimate of the hyperparameter. This mixed approach is usually referred to as empirical Bayes. Since the prior density (8) belongs to a parametric family with unknown prior hyperparameter $b$, such parameter is to be estimated using the available information. In theory, the accuracy of maximum likelihood estimation is higher than the moment estimation. In view of this fact, we will use maximum likelihood method to estimate the hyperparameter of $b$ using the marginal probability density function:

$$
\begin{aligned}
m(\underline{x} \mid b) & =\int_{0}^{\infty} L(\underline{x} \mid \theta) \pi(\theta \mid b) d \theta \\
& =\frac{A \Gamma(b+m) 2^{m} \prod_{i=1}^{m} x_{(i)}}{\Gamma(b)(1+T)^{b+m}} .
\end{aligned}
$$

Based on (16), we obtain the likelihood equation in the form

$$
\ln \left[\frac{b+m}{b(1+T)}\right]+\frac{m}{2 b(b+m)}=0 .
$$

Equation (17) has no closed form solution in $b$. Therefore, we obtain the estimate $\widehat{b}$ for $b$ by solving the equation via numerical method. Following the idea of Lehmann and Casella [9], we can obtain the EB estimates $\widehat{\theta}_{\mathrm{EGE}}, \widehat{R}(t)_{\mathrm{EGE}}$, and $\widehat{\lambda}(t)_{\mathrm{EGE}}$ by substituting $\widehat{b}$ for $b$ in (15).
2.6. Risk Functions of Estimates under GELF. At this point, we obtain the risk functions of EB and GML estimates of reliability performances under GELF. The risk function of an estimate $\widehat{\phi}$ under GELF is given by

$$
\begin{aligned}
R_{L}(\widehat{\phi}) & =E^{h}[L(\widehat{\phi}, \phi)] \\
& =\int_{0}^{\infty}\left[\left(\frac{\hat{\phi}}{\phi}\right)^{\alpha}-\alpha \ln \left(\frac{\hat{\phi}}{\phi}\right)-1\right] h(T) d T .
\end{aligned}
$$

The risk functions of $\widehat{\theta}_{\mathrm{EGE}}$ and $\widehat{\theta}_{\mathrm{GML}}$ under GELF are given, respectively, by

$$
\begin{aligned}
& R_{L}\left(\widehat{\theta}_{\mathrm{EGE}}\right) \\
& =\left\{\begin{array}{l}
\frac{1}{\Gamma(m)}\left[\frac{\Gamma(\widehat{b}+m)}{2^{\alpha / 2} \theta^{\alpha} \Gamma(\widehat{b}+m+\alpha / 2)} S_{1}-\frac{\alpha}{2} S_{3}\right]+v_{1}-1, \\
\text { when } T<1, \\
\frac{1}{\Gamma(m)}\left[\frac{\Gamma(\widehat{b}+m)}{\Gamma(\widehat{b}+m+\alpha / 2)} S_{2}-\frac{\alpha}{2} S_{4}\right]+v_{2}+\frac{\alpha}{4 m}-1, \\
\text { when } T>1,
\end{array}\right. \\
& R_{L}\left(\widehat{\theta}_{\mathrm{GML}}\right) \\
& \left\{\frac{1}{\Gamma(m)}\left\{\frac{1}{(\sqrt{2 \widehat{b}+2 m+1} \theta)^{\alpha}} S_{1}-\frac{\alpha}{2} S_{3}\right\}\right. \\
& +\alpha \ln (\sqrt{2 \widehat{b}+2 m+1} \theta)-1 \text {, } \\
& =\{\text { when } T<1 \text {, } \\
& \frac{1}{\Gamma(m)}\left\{\left(\widehat{b}+m+\frac{1}{2}\right)^{-\alpha / 2} S_{2}-\frac{\alpha}{2} S_{4}\right\} \\
& +\frac{\alpha}{2}\left[\ln \left(1+\frac{2 \widehat{b}+1}{2 m}\right)+\frac{1}{2 m}\right]-1, \\
& \text { when } T>1 \text {, }
\end{aligned}
$$

where

$$
\begin{aligned}
& S_{1}=\sum_{k=0}^{\infty}\left(\begin{array}{l}
\frac{\alpha}{2} \\
k
\end{array}\right)\left(2 \theta^{2}\right)^{k} \Gamma(m+k) ; \\
& S_{2}=\sum_{k=0}^{\infty}\left(\begin{array}{l}
\frac{\alpha}{2} \\
k
\end{array}\right)\left(2 \theta^{2}\right)^{-k} \Gamma\left(m-k+\frac{\alpha}{2}\right) ; \\
& S_{3}=\sum_{k=1}^{\infty} \frac{(-1)^{k+1}\left(2 \theta^{2}\right)^{k} \Gamma(m+k)}{k} ; \\
& S_{4}=\sum_{k=1}^{\infty} \frac{(-1)^{k+1}\left(2 \theta^{2}\right)^{-k} \Gamma(m-k)}{k} ;
\end{aligned}
$$




$$
\begin{aligned}
& v_{1}=\ln \left[\frac{\Gamma(\widehat{b}+m+\alpha / 2) 2^{\alpha / 2} \theta^{\alpha}}{\Gamma(\widehat{b}+m)}\right] ; \\
& v_{2}=\ln \left[\frac{\Gamma(\widehat{b}+m+\alpha / 2)}{m^{\alpha / 2} \Gamma(\widehat{b}+m)}\right] .
\end{aligned}
$$

The risk functions of $\widehat{R}(t)_{\mathrm{EGE}}$ and $\widehat{R}(t)_{\mathrm{GML}}$ under GELF are given, respectively, by

$$
\begin{aligned}
& R_{L}\left(\widehat{R}(t)_{\mathrm{EGE}}\right) \\
& =\left\{\begin{array}{l}
\frac{1}{\Gamma(m)}\left[\exp \left(\frac{t^{2} \alpha}{2 \theta^{2}}\right) R_{1}+(\widehat{b}+m) R_{3}\right]-\frac{t^{2} \alpha}{2 \theta^{2}}-1, \\
\text { when } T<1 \\
\frac{1}{\Gamma(m)}\left[\exp \left(\frac{t^{2} \alpha}{2 \theta^{2}}\right) R_{2}+(\widehat{b}+m) R_{4}\right]-\frac{t^{2} \alpha}{2 \theta^{2}}-1, \\
\quad \text { when } T>1,
\end{array}\right. \\
& \text { if }-1 \leq \frac{t^{2} \alpha}{1+T}<1 \\
& R_{L}\left(\widehat{R}(t)_{\mathrm{GML}}\right) \\
& =\left\{\begin{array}{l}
\frac{1}{\Gamma(m)}\left[\exp \left(\frac{t^{2} \alpha}{2 \theta^{2}}\right) R_{5}+\frac{\alpha(2 \widehat{b}+2 m+1) t^{2}}{2} S_{5}\right] \\
-\frac{t^{2} \alpha}{2 \theta^{2}}-1, \\
\text { when } T<1, \\
\frac{1}{\Gamma(m)}\left[\exp \left(\frac{t^{2} \alpha}{2 \theta^{2}}\right) R_{6}+\frac{\alpha(2 \widehat{b}+2 m+1) t^{2}}{4 \theta^{2}} S_{6}\right] \\
-\frac{t^{2} \alpha}{2 \theta^{2}}-1, \\
\text { when } T>1,
\end{array}\right.
\end{aligned}
$$

where

$$
\begin{aligned}
& R_{1}=\sum_{k=0}^{\infty} \sum_{p=0}^{\infty}\left(\begin{array}{c}
\widehat{b}+m \\
k
\end{array}\right)\left(\begin{array}{c}
-k \\
p
\end{array}\right)\left(-t^{2} \alpha\right)^{k}\left(2 \theta^{2}\right)^{p} \Gamma(m+p) ; \\
& R_{2}=\sum_{k=0}^{\infty} \sum_{p=0}^{\infty}\left(\begin{array}{c}
\widehat{b}+m \\
k
\end{array}\right)\left(\begin{array}{c}
-k \\
p
\end{array}\right)\left(-t^{2} \alpha\right)^{k} \\
& \quad \times\left(2 \theta^{2}\right)^{-(k+p)} \Gamma(m-p-k) ; \\
& R_{3}=\sum_{k=1}^{\infty} \sum_{p=0}^{\infty}\left(\begin{array}{c}
-k \\
p
\end{array}\right)\left(t^{2} \alpha\right)^{k} k^{-1}\left(2 \theta^{2}\right)^{p} \Gamma(m+p) ; \\
& R_{4}=\sum_{k=1}^{\infty} \sum_{p=0}^{\infty}\left(\begin{array}{c}
-k \\
p
\end{array}\right)\left(t^{2} \alpha\right)^{k} k^{-1}\left(2 \theta^{2}\right)^{-(k+p)} \Gamma(m-p-k) ;
\end{aligned}
$$

$$
\begin{gathered}
R_{5}=\sum_{k=0}^{\infty} \sum_{p=0}^{\infty}\left(\begin{array}{c}
-k \\
p
\end{array}\right)\left(-\frac{\alpha(2 \widehat{b}+2 m+1) t^{2}}{2}\right)^{k} \\
\times\left(\frac{\Gamma(m+p)}{\Gamma(k+1)}\right)\left(2 \theta^{2}\right)^{p} ; \\
R_{6}=\sum_{k=0}^{\infty} \sum_{p=0}^{\infty}\left(\begin{array}{c}
-k \\
p
\end{array}\right)\left(-\frac{\alpha(2 \widehat{b}+2 m+1) t^{2}}{2}\right)^{k} \\
\quad \times\left(\frac{\Gamma(m-k-p)}{\Gamma(k+1)}\right)\left(2 \theta^{2}\right)^{-(k+p)} ; \\
S_{5}=\sum_{k=0}^{\infty}\left(\begin{array}{c}
-1 \\
k
\end{array}\right)\left(2 \theta^{2}\right)^{k} \Gamma(m+k) ; \\
S_{6}=\sum_{k=0}^{\infty}\left(\begin{array}{c}
-1 \\
k
\end{array}\right)\left(2 \theta^{2}\right)^{-k} \Gamma(m-k-1) .
\end{gathered}
$$

The risk functions of $\hat{\lambda}(t)_{\mathrm{EGE}}$ and $\hat{\lambda}(t)_{\mathrm{GML}}$ under GELF are given, respectively, by

$$
\begin{aligned}
& R_{L}\left(\hat{\lambda}(t)_{\mathrm{EGE}}\right) \\
& =\left\{\begin{array}{l}
\frac{1}{\Gamma(m)}\left[\frac{\left(2 \theta^{2}\right)^{\alpha} \Gamma(\widehat{b}+m)}{\Gamma(\widehat{b}+m-\alpha)} S_{7}+\alpha S_{3}\right]+v_{5}-1, \\
\text { when } T<1, \\
\frac{1}{\Gamma(m)}\left[\frac{\Gamma(\widehat{b}+m)}{\Gamma(\widehat{b}+m-\alpha)} S_{8}+\alpha S_{4}\right]+v_{6}-\frac{\alpha}{2 m}-1, \\
\text { when } T>1,
\end{array}\right. \\
& R_{L}\left(\hat{\lambda}(t)_{\mathrm{GML}}\right) \\
& \left\{\begin{array}{l}
\frac{1}{\Gamma(m)}\left\{\left[(2 \widehat{b}+2 m+1) \theta^{2}\right]^{\alpha} S_{7}+\alpha S_{3}\right\} \\
\quad-\alpha \ln \left[(2 \widehat{b}+2 m+1) \theta^{2}\right]-1, \\
\text { when } T<1 \\
\frac{1}{\Gamma(m)}\left[\left(\widehat{b}+m+\frac{1}{2}\right)^{\alpha} S_{8}+\alpha S_{4}\right] \\
-\alpha\left[\ln \left(1+\frac{2 \widehat{b}+1}{2 m}\right)+\frac{1}{2 m}\right]-1,
\end{array}\right. \\
& \text { when } T>1 \text {, }
\end{aligned}
$$

where

$$
\begin{aligned}
& S_{7}=\sum_{k=0}^{\infty}\left(\begin{array}{c}
-\alpha \\
k
\end{array}\right)\left(2 \theta^{2}\right)^{k} \Gamma(m+k) ; \\
& S_{8}=\sum_{k=0}^{\infty}\left(\begin{array}{c}
-\alpha \\
k
\end{array}\right)\left(2 \theta^{2}\right)^{-k} \Gamma(m-k-\alpha) ; \\
& v_{5}=\ln \left[\frac{\Gamma(\widehat{b}+m-\alpha)}{\Gamma(\widehat{b}+m)\left(2 \theta^{2}\right)^{\alpha}}\right] \\
& v_{6}=\ln \left[\frac{\Gamma(\widehat{b}+m-\alpha)}{\Gamma(\widehat{b}+m)} m^{\alpha}\right]
\end{aligned}
$$


TABLE 1: Progressively Type II censoring schemes (C.S.) applied in the simulation study $(b=25, m=35$, and $t=0.04)$.

\begin{tabular}{lcc}
\hline$n$ & C.S. & $\underline{r}=\left(r_{1}, r_{2}, \ldots, r_{m}\right)$ \\
\hline \multirow{2}{*}{100} & {$[1]$} & $(2 * 16,1 * 2,2 * 16)$ \\
& {$[2]$} & $(0 * 33,66)$ \\
\hline \multirow{2}{*}{90} & {$[3]$} & $(2 * 14,0 * 6,2 * 14)$ \\
& {$[4]$} & $(0 * 33,56)$ \\
\hline \multirow{2}{*}{80} & {$[5]$} & $(2 * 11,1,0 * 10,1,2 * 11)$ \\
& {$[6]$} & $(0 * 33,46)$ \\
\hline 70 & {$[7]$} & $(0 * 33,36)$ \\
\hline \multirow{2}{*}{60} & {$[8]$} & $(1 * 13,0 * 8,1 * 13)$ \\
& {$[9]$} & $(0 * 33,26)$ \\
\hline
\end{tabular}

TABLE 2: GML and EB estimates of parameter $\theta$ for different values of $\alpha$.

\begin{tabular}{lccccc}
\hline C.S. & $\hat{\theta}_{\mathrm{GML}}$ & $\alpha=-0.8$ & $\alpha=-0.5$ & $\alpha=0.5$ & $\alpha=0.8$ \\
\hline$[1]$ & 0.15953 & 0.16132 & 0.16121 & 0.16083 & 0.16071 \\
{$[2]$} & 0.14362 & 0.14511 & 0.14501 & 0.14470 & 0.14460 \\
{$[3]$} & 0.16136 & 0.16319 & 0.16308 & 0.16269 & 0.16257 \\
{$[4]$} & 0.14498 & 0.14649 & 0.14640 & 0.14608 & 0.14598 \\
{$[5]$} & 0.16401 & 0.16590 & 0.16578 & 0.16538 & 0.16526 \\
{$[6]$} & 0.14690 & 0.14845 & 0.14835 & 0.14802 & 0.14793 \\
{$[7]$} & 0.16816 & 0.17012 & 0.17000 & 0.16959 & 0.16946 \\
{$[8]$} & 0.14980 & 0.15141 & 0.15131 & 0.15097 & 0.15087 \\
{$[9]$} & 0.18264 & 0.18488 & 0.18474 & 0.18426 & 0.18412 \\
{$[10]$} & 0.15473 & 0.15643 & 0.15633 & 0.15597 & 0.15586 \\
\hline
\end{tabular}

2.7. Risk Efficiencies under GELF. The risk efficiency of an estimate $\widehat{\phi}_{1}$ with respect to an estimate $\widehat{\phi}_{2}$ under GELF is denoted by $\operatorname{RE}_{L}\left(\widehat{\phi}_{1}, \widehat{\phi}_{2}\right)$ and can be defined as

$$
\operatorname{RE}_{L}\left(\widehat{\phi}_{1}, \widehat{\phi}_{2}\right)=\frac{R_{L}\left(\widehat{\phi}_{2}\right)}{R_{L}\left(\widehat{\phi}_{1}\right)}
$$

Under GELF, the risk efficiencies $\mathrm{RE}_{L}\left(\widehat{\theta}_{\mathrm{EGE}}, \widehat{\theta}_{\mathrm{GML}}\right)$, $\mathrm{RE}_{L}\left(\widehat{R}(t)_{\mathrm{EGE}}, \widehat{R}(t)_{\mathrm{GML}}\right)$, and $\mathrm{RE}_{L}\left(\widehat{\lambda}(t)_{\mathrm{EGE}}, \widehat{\lambda}(t)_{\mathrm{GML}}\right)$ can be obtained, respectively, by using the results (19)-(23) in (25).

\section{Results and Discussion}

In this section, the performance of EB and GML estimates has been examined and compared on the basis of an extensive Monte Carlo simulation study and real life data application.

3.1. A Simulation Study. This subsection considers an extensive Monte Carlo simulation study to examine and compare the GML and EB estimates of reliability performances. The comparison is made on the basis of the risk efficiency criteria for different sample sizes $(n=60,70,80,90,100)$. The risk efficiencies under GELF were computed to see whether the EB estimates relative to GELF outperform the GML estimates or not. As one data set does not help to clarify the performance of the estimate, we have computed the estimates and risk efficiencies by averaging the estimates and risk efficiencies (at $t=0.04$ ) over 2,000 simulations of progressively Type II censored samples of size $m$. These samples were generated from the Rayleigh distribution according to the algorithm given in Balakrishnan and Sandhu [14].

Table 1 represents the different progressive Type II censoring scheme considered in the study. The simulated results are reported in Tables 2, 3, 4, 5, 6, and 7, respectively. From the simulation results, the following points can be drawn.

(1) The risk efficiencies $\mathrm{RE}_{L}\left(\widehat{\theta}_{\mathrm{EGE}}, \widehat{\theta}_{\mathrm{GML}}\right), \mathrm{RE}_{L}\left(\widehat{R}(t)_{\mathrm{EGE}}\right.$, $\left.\widehat{R}(t)_{\mathrm{GML}}\right)$, and $\mathrm{RE}_{L}\left(\widehat{\lambda}(t)_{\mathrm{EGE}}, \widehat{\lambda}(t)_{\mathrm{GML}}\right)$ are greater than one for all the considered cases, which indicates that the proposed EB estimates of reliability performances are more preferable than the GML estimates (see Tables 3, 5, and 7).

(2) As the effective sample proportion $m / n$ increases, the risk efficiencies $\mathrm{RE}_{L}\left(\widehat{\theta}_{\mathrm{EGE}}, \hat{\theta}_{\mathrm{GML}}\right)$ and $\mathrm{RE}_{\mathrm{L}}\left(\widehat{\lambda}(t)_{\mathrm{EGE}}\right.$, $\left.\widehat{\lambda}(t)_{\mathrm{GML}}\right)$ increase, while $\mathrm{RE}_{L}\left(\widehat{R}(t)_{\mathrm{EGE}}, \widehat{R}(t)_{\mathrm{GML}}\right)$ decreases (see Tables 3, 7, and 5).

(3) The risk efficiencies $\operatorname{RE}_{L}\left(\hat{\theta}_{\mathrm{EGE}}, \widehat{\theta}_{\mathrm{GML}}\right)$ and $\mathrm{RE}_{L}\left(\widehat{\lambda}(t)_{\mathrm{EGE}}, \hat{\lambda}(t)_{\mathrm{GML}}\right)$ for the progressive Type II samples are greater than those for the conventional Type II samples (see Tables 3 and 7).

(4) The risk efficiencies $\operatorname{RE}_{L}\left(\widehat{R}(t)_{\mathrm{EGE}}, \widehat{R}(t)_{\mathrm{GML}}\right)$ for the progressive Type II samples are smaller than those for the conventional Type II samples (see Table 5).

(5) The risk efficiencies under GELF are very sensitive to variation in " $\alpha$ " (see Tables 3,5 , and 7).

(6) Different values of the hyperparameter $b$ have been examined, and almost the same conclusions stated above are followed. It may be mentioned here that because of space restriction, results for all the variations are not shown.

3.2. Real Data Application. We have considered the wind speed data set of Elanora Heights, located at northeastern suburb of Sydney, Australia [15], by making some modification for comparing the performance of proposed EB estimates with that of GML estimates in terms of risk efficiency. The average daily wind speeds (in meter/sec) reported during November 2007 were as follows: 
TABLE 3: Risk efficiency of $\widehat{\theta}_{\mathrm{EGE}}$ with respect to $\widehat{\theta}_{\mathrm{GML}}$ under GELF for different values of $\alpha$.

\begin{tabular}{lcccc}
\hline \multirow{2}{*}{ C.S. } & $\alpha=-0.8$ & $\alpha=-0.5$ & $\alpha=0.5$ & $\alpha=0.8$ \\
\hline$[1]$ & 1.01499 & 1.01316 & 1.01300 & 1.01480 \\
{$[2]$} & 1.01132 & 1.00885 & 1.00477 & 1.00474 \\
{$[3]$} & 1.01542 & 1.01367 & 1.01400 & 1.01607 \\
{$[4]$} & 1.01162 & 1.00920 & 1.00543 & 1.00552 \\
{$[5]$} & 1.01604 & 1.01440 & 1.01548 & 1.01793 \\
{$[6]$} & 1.01206 & 1.00972 & 1.00638 & 1.00668 \\
{$[7]$} & 1.01699 & 1.01555 & 1.01783 & 1.02092 \\
{$[8]$} & 1.01272 & 1.01049 & 1.00782 & 1.00843 \\
{$[9]$} & 1.02027 & 1.01949 & 1.02642 & 1.03227 \\
{$[10]$} & 1.01387 & 1.01184 & 1.01041 & 1.01160 \\
\hline
\end{tabular}

TABLE 4: GML and EB estimates of reliability $R(t)$ for different values of $\alpha$.

\begin{tabular}{lccccc}
\hline \multirow{2}{*}{ C.S. } & $\widehat{R}(t)_{\mathrm{GML}}$ & $\alpha=-0.8$ & $\alpha=-0.5$ & $\alpha=0.5$ & $\alpha=0.8$ \\
\hline$[1]$ & 0.96837 & 0.96866 & 0.96865 & 0.96865 & 0.96864 \\
{$[2]$} & 0.96112 & 0.96145 & 0.96145 & 0.96143 & 0.96143 \\
{$[3]$} & 0.96907 & 0.96936 & 0.96935 & 0.96934 & 0.96934 \\
{$[4]$} & 0.96183 & 0.96216 & 0.96216 & 0.96214 & 0.96214 \\
{$[5]$} & 0.97004 & 0.97032 & 0.97032 & 0.97031 & 0.97031 \\
{$[6]$} & 0.96280 & 0.96312 & 0.96312 & 0.96311 & 0.96310 \\
{$[7]$} & 0.97148 & 0.97175 & 0.97174 & 0.97174 & 0.97173 \\
{$[8]$} & 0.96420 & 0.96452 & 0.96452 & 0.96450 & 0.96450 \\
{$[9]$} & 0.97576 & 0.97600 & 0.97600 & 0.97600 & 0.97599 \\
{$[10]$} & 0.96640 & 0.96670 & 0.96670 & 0.96669 & 0.96669 \\
\hline
\end{tabular}

TABLE 5: Risk efficiency of $\widehat{R}(t)_{\mathrm{EGE}}$ with respect to $\widehat{R}(t)_{\mathrm{GML}}$ under GELF for different values of $\alpha$.

\begin{tabular}{lcccc}
\hline \multirow{2}{*}{ C.S. } & \multicolumn{4}{c}{$\mathrm{RE}_{L}\left(\widehat{R}(t)_{\mathrm{EGE}}, \widehat{R}(t)_{\mathrm{GML}}\right)$} \\
& $\alpha=-0.8$ & $\alpha=-0.5$ & $\alpha=0.5$ & $\alpha=0.8$ \\
\hline$[1]$ & 1.00505 & 1.00151 & 1.00017 & 1.00084 \\
{$[2]$} & 1.00622 & 1.00213 & 1.00026 & 1.00096 \\
{$[3]$} & 1.00498 & 1.00148 & 1.00016 & 1.00075 \\
{$[4]$} & 1.00605 & 1.00202 & 1.00024 & 1.00086 \\
{$[5]$} & 1.00488 & 1.00145 & 1.00014 & 1.00067 \\
{$[6]$} & 1.00588 & 1.00192 & 1.00022 & 1.00077 \\
{$[7]$} & 1.00473 & 1.00141 & 1.00011 & 1.00059 \\
{$[8]$} & 1.00557 & 1.00172 & 1.00018 & 1.00068 \\
{$[9]$} & 1.00430 & 1.00127 & 1.00009 & 1.00045 \\
{$[10]$} & 1.00528 & 1.00159 & 1.00017 & 1.00051 \\
\hline
\end{tabular}

We have checked the validity of the Rayleigh model based on the estimated value (moment estimate) of parameter $\theta=$ 0.9242 , using two different measures, Kolmogorov-Smirnov (KS) test, and Anderson-Darling (AD) test. The KS test (test statistic value $=0.20475$ with $P$ value 0.14012 and critical value $=0.2417)$ and $\mathrm{AD}$ test (test statistic $=1.9547$ with critical value $=2.5018$ ) suggest that one-parameter Rayleigh distribution provides an adequate fit to this data set.

As a numerical illustration, we have generated artificial progressive and conventional Type II censored samples of
TABLE 6: GML and EB estimates of failure rate $\lambda(t)$ for different values of $\alpha$.

\begin{tabular}{lccccc}
\hline C.S. & $\hat{\lambda}(t)_{\mathrm{GML}}$ & $\alpha=-0.8$ & $\alpha=-0.5$ & $\alpha=0.5$ & $\alpha=0.8$ \\
\hline$[1]$ & 1.60797 & 1.59047 & 1.58611 & 1.57152 & 1.56714 \\
{$[2]$} & 1.98391 & 1.96402 & 1.95906 & 1.94248 & 1.93749 \\
{$[3]$} & 1.57169 & 1.55445 & 1.55014 & 1.53577 & 1.53145 \\
{$[4]$} & 1.94688 & 1.92721 & 1.92230 & 1.90590 & 1.90097 \\
{$[5]$} & 1.52142 & 1.50454 & 1.50033 & 1.48625 & 1.48202 \\
{$[6]$} & 1.89643 & 1.87707 & 1.87223 & 1.85609 & 1.85124 \\
{$[7]$} & 1.44761 & 1.01774 & 1.42718 & 1.41356 & 1.40946 \\
{$[8]$} & 1.82369 & 1.80477 & 1.80005 & 1.78428 & 1.77954 \\
{$[9]$} & 1.22733 & 1.21271 & 1.20906 & 1.19688 & 1.19322 \\
{$[10]$} & 1.70965 & 1.69147 & 1.68693 & 1.67177 & 1.66722 \\
\hline
\end{tabular}

TABLE 7: Risk efficiency of $\hat{\lambda}(t)_{\mathrm{EGE}}$ with respect to $\hat{\lambda}(t)_{\mathrm{GML}}$ under GELF for different values of $\alpha$.

\begin{tabular}{lcccc}
\hline \multirow{2}{*}{ C.S. } & \multicolumn{4}{c}{$\mathrm{RE}_{L}\left(\hat{\lambda}(t)_{\mathrm{EGE}}, \widehat{\lambda}(t)_{\mathrm{GML}}\right)$} \\
\hline$[1]$ & $\alpha=-0.8$ & $\alpha=-0.5$ & $\alpha=0.5$ & $\alpha=0.8$ \\
\hline$[2]$ & 1.02079 & 1.01620 & 1.01666 & 1.02375 \\
{$[3]$} & 1.02288 & 1.01765 & 1.01705 & 1.02409 \\
{$[4]$} & 1.00670 & 1.00573 & 1.01357 & 1.02100 \\
{$[5]$} & 1.02306 & 1.01980 & 1.01761 & 1.02457 \\
{$[6]$} & 1.00836 & 1.00702 & 1.01397 & 1.02137 \\
{$[7]$} & 1.02443 & 1.02328 & 1.01848 & 1.02532 \\
{$[8]$} & 1.01092 & 1.00898 & 1.01458 & 1.02192 \\
{$[9]$} & 1.02567 & 1.02690 & 1.02143 & 1.02780 \\
{$[10]$} & 1.01571 & 1.01255 & 1.01563 & 1.02286 \\
\hline
\end{tabular}

size $m=20$ from this data set. In the first case, we have considered the vector of observed failure times $\underline{x}=(0.5833$, $0.6667,0.7222,0.7500,0.7778,0.8056,0.8889,0.9167,1.0000$, $1.0278,1.1111,1.1111,1.1667,1.1944,1.2778,1.3333,1.3611,1.4444$, $2.1389,2.7778)$ with the progressive Type II censoring scheme $\underline{r}=(1 * 5,0 * 10,1 * 5)$. In the second case, we have considered the vector of observed failure times $x=(0.5833,0.6667$, $0.6944,0.7222,0.7500,0.7778,0.8056,0.8056,0.8611,0.8889$, $0.9167,1.0000,1.0278,1.0278,1.1111,1.1111,1.1111,1.1667,1.1667$, 1.1944) with the conventional Type II censoring scheme $\underline{r}=$ $(0 * 19,10)$. For these samples, the risk efficiencies of the estimates of reliability performances (at $t=0.04$ ) under GELF were computed using the results outlined in Section 2 and reported in Tables 8-9.

From Tables 8-9, it is observed that the risk efficiencies $\operatorname{RE}_{L}\left(\widehat{\theta}_{\mathrm{EGE}}, \widehat{\theta}_{\mathrm{GML}}\right), \quad \mathrm{RE}_{L}\left(\widehat{R}(t)_{\mathrm{EGE}}, \widehat{R}(t)_{\mathrm{GML}}\right)$, and $\mathrm{RE}_{L}\left(\widehat{\lambda}(t)_{\mathrm{EGE}}, \widehat{\lambda}(t)_{\mathrm{GML}}\right)$ are greater than one for all values of $\alpha(\alpha= \pm 0.5, \pm 0.8)$. This indicates that the proposed EB estimates are more preferable than GML estimates in both considered cases. 
TABle 8: The estimates and risk efficiencies for the progressive Type II censored data.

\begin{tabular}{lcccccc}
\hline \multicolumn{6}{c}{$\hat{\theta}_{\mathrm{GML}}=1.11883 ; \widehat{R}(t)_{\mathrm{GML}}=0.99936 ; \hat{\lambda}(t)_{\mathrm{GML}}=0.03195$} \\
$\alpha$ & $\hat{\theta}_{\mathrm{EGE}}$ & $\mathrm{RE}_{L}\left(\widehat{\theta}_{\mathrm{EGE}}, \widehat{\theta}_{\mathrm{GML}}\right)$ & $\widehat{R}(t)_{\mathrm{EGE}}$ & $\mathrm{RE}_{L}\left(\widehat{R}(t)_{\mathrm{EGE}}, \widehat{R}(t)_{\mathrm{GML}}\right)$ & $\hat{\lambda}(t)_{\mathrm{EGE}}$ & $\mathrm{RE}_{L}\left(\widehat{\lambda}(t)_{\mathrm{EGE}}, \hat{\lambda}(t)_{\mathrm{GML}}\right)$ \\
\hline-0.8 & 1.15179 & 1.08914 & 0.99937 & 1.00004 & 0.03105 & 1.02717 \\
-0.5 & 1.14963 & 1.08625 & 0.99937 & 1.00003 & 0.03083 & 1.04094 \\
0.5 & 1.14256 & 1.06394 & 0.99937 & 1.00001 & 0.03007 & 1.09466 \\
0.8 & 1.14046 & 1.04728 & 0.99937 & 1.00005 & 0.02985 & 1.11571 \\
\hline
\end{tabular}

TABle 9: The estimates and risk efficiencies for the conventional Type II censored data.

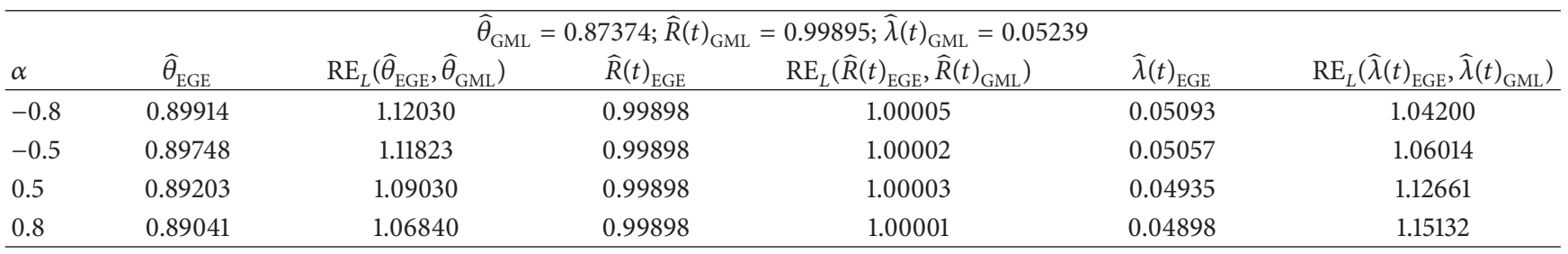

\section{Conclusion}

This paper is devoted to the comparative study of the performance of EB and GML estimates of reliability performances. We have obtained the GML estimates and EB estimates under GELF when the data are progressively Type II censored from the Rayleigh distribution. These estimates are then compared using the risk efficiency criteria. We have presented the real data example to illustrate the proposed estimation methods. A Monte Carlo simulation study is carried out to compare the performance of EB estimates with that of GML estimates. The study demonstrates that the EB estimates under GELF exhibit better performance than the GML estimates. Moreover, the risk efficiencies get smaller with the increasing ratio $m / n$. Based on the present study, the EB estimation is recommended for the estimation of reliability performances of the Rayleigh model.

\section{Conflict of Interests}

The authors declare that there is no conflict of interests regarding the publication of this paper.

\section{Acknowledgments}

The authors are thankful to the honorable editor and anonymous reviewers for valuable comments and constructive suggestions, which significantly improved the paper.

\section{References}

[1] A. C. Cohen, "Progressively censored samples in life testing," Technometrics, vol. 5, pp. 327-339, 1963.

[2] N. Balakrishnan and R. Aggarwala, Progressive CensoringTheory, Methods and Applications, Statistics for Industry and Technology, Birkhäuser, Boston, Mass, USA, 2000.

[3] S. Wu, D. Chen, and S. Chen, "Bayesian inference for Rayleigh distribution under progressive censored sample," Applied Stochastic Models in Business and Industry, vol. 22, no. 3, pp. 269-279, 2006.
[4] N. W. Patel and M. N. Patel, "Progressively type-II censored sample from geometric life time model," Journal of Probability and Statistical Science, vol. 5, no. 1, pp. 81-95, 2007.

[5] H. Robbins, "The empirical Bayes approach to statistical decision problems," Annals of Mathematical Statistics, vol. 35, no. 1, pp. 1-20, 1964.

[6] J. S. Maritz and T. Lwin, Empirical Bayes Methods, vol. 35 of Monographs on Statistics and Applied Probability, Chapman \& Hall, London, UK, 2nd edition, 1989.

[7] G. Casella, "Illustrating empirical Bayes methods," Chemometrics and Intelligent Laboratory Systems, vol. 16, no. 2, pp. 107-125, 1992.

[8] B. P. Carlin and T. A. Louis, Bayes and Empirical Bayes Methods for Data Analysis, Chapman \& Hall, London, UK, 1996.

[9] E. L. Lehmann and G. Casella, Theory of Point Estimation, Springer, Second edition, 1998.

[10] M. A. M. Ali Mousa, "Empirical Bayes estimators for the Burr type XII accelerated life-testing model based on Type II censored data," Journal of Statistical Computation and Simulation, vol. 52, pp. 95-103, 1995.

[11] A. Asgharzadeh and R. Valiollahi, "Estimation based on progressively censored data from the Burr model," International Mathematical Forum, vol. 3, no. 43, pp. 2113-2121, 2008.

[12] L. Rayleigh, "On the resultant of a large number of vibrations of same pitch and of arbitrary phase," Philosophical Magazine, vol. 10 , no. 60 , pp. $73-78,1880$.

[13] R. Calabria and G. Pulcini, "An engineering approach to Bayes estimation for the Weibull distribution," Microelectronics Reliability, vol. 34, no. 5, pp. 789-802, 1994.

[14] N. Balakrishnan and R. A. Sandhu, "A simple simulational algorithm for generating progressive type-II censored samples," The American Statistician, vol. 49, no. 2, pp. 229-230, 1995.

[15] D. J. Best, J. C. W. Rayner, and O. Thas, "Easily applied tests of fit for the Rayleigh distribution," Sankhya B, vol. 72, no. 2, pp. 254-263, 2010. 


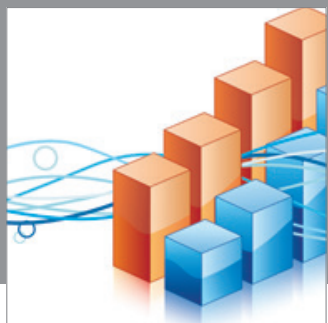

Advances in

Operations Research

mansans

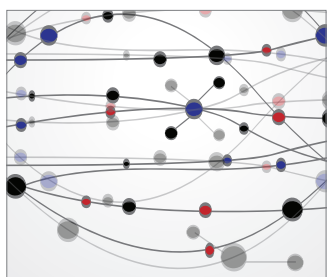

The Scientific World Journal
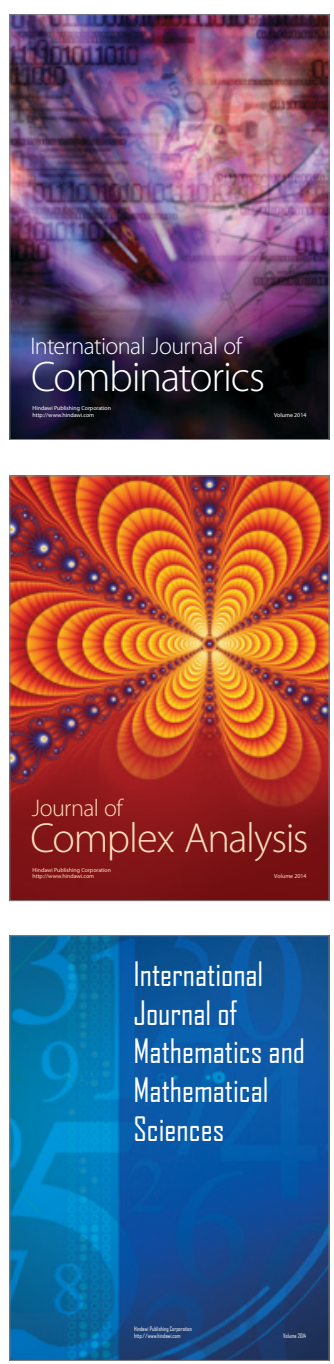
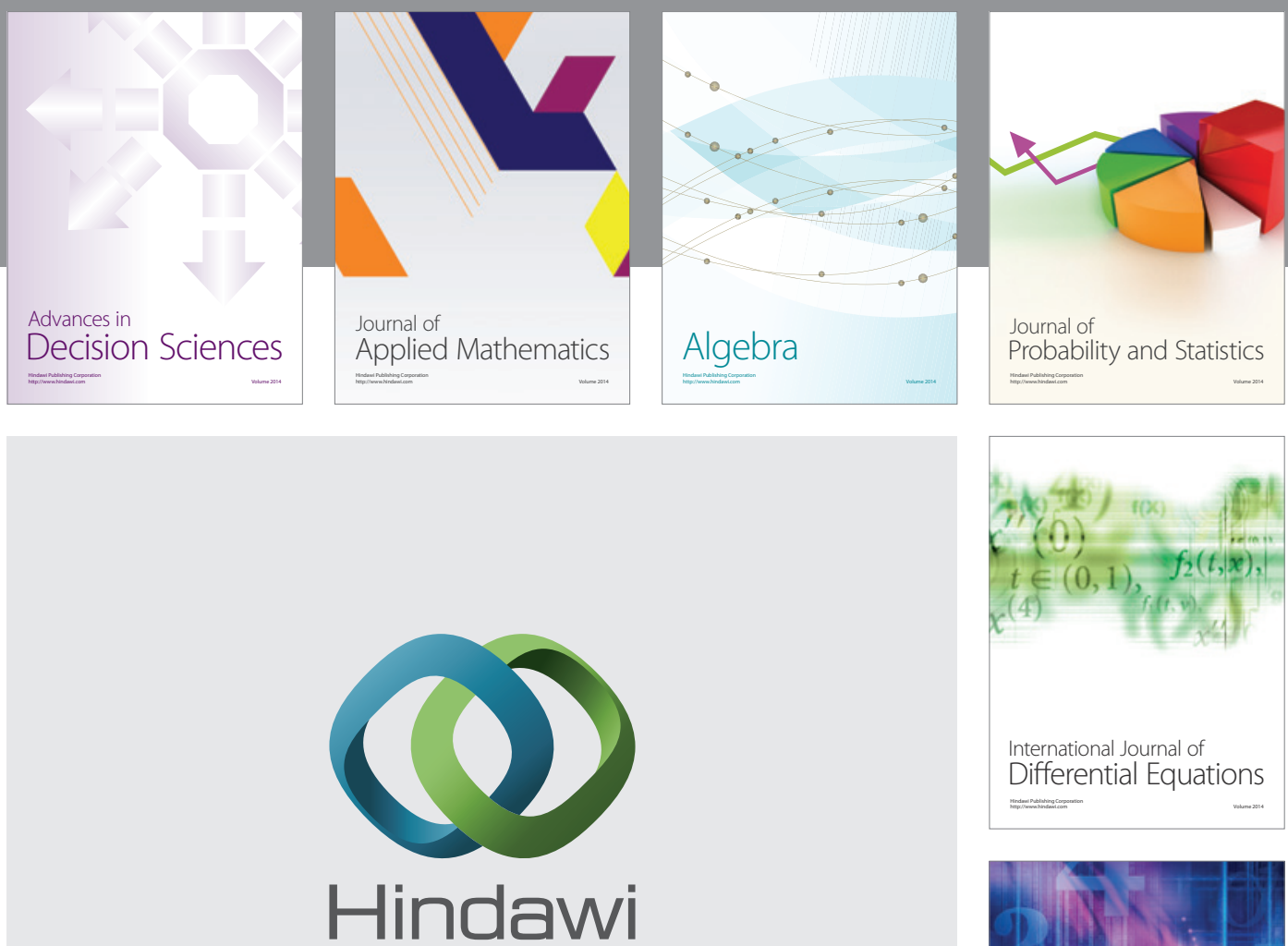

Submit your manuscripts at http://www.hindawi.com
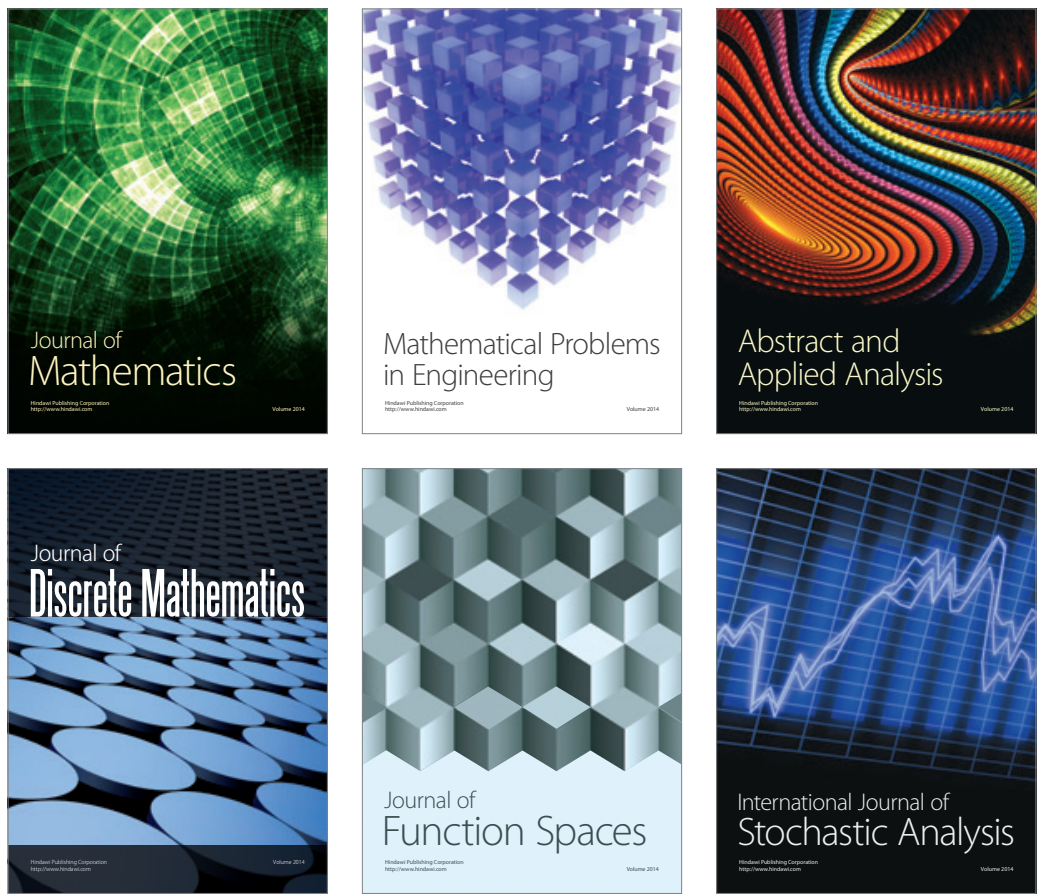

Journal of

Function Spaces

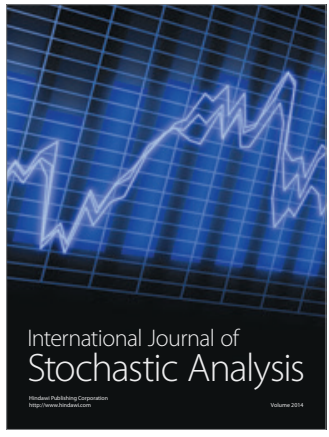

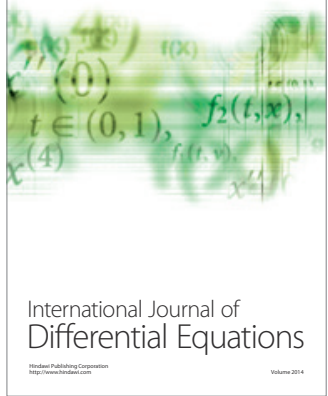
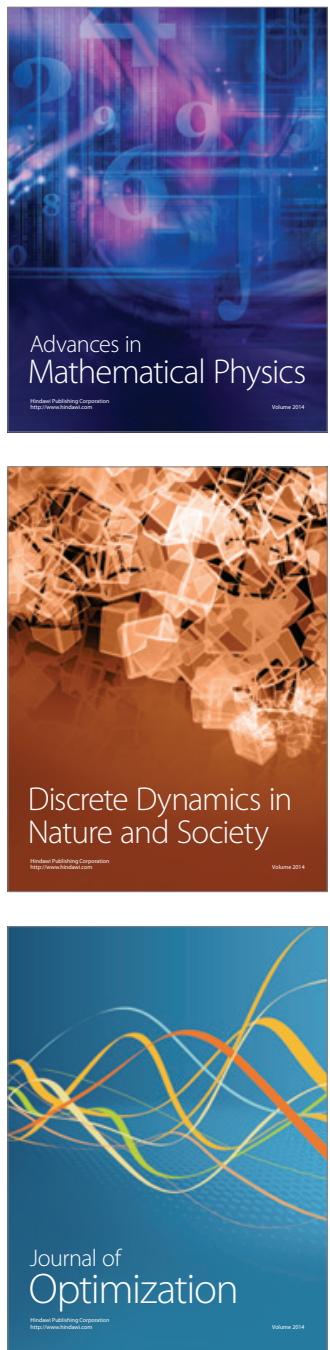